\title{
Hydrodynamical evolution near the QCD critical end point
}

\author{
Chiho Nonaka \\ Department of Physics, Duke University, Durham, NC 27ro8, U.S.A. \\ Masayuki Asakawa \\ Department of Physics, Osaka University, Toyonaka 560-0043, Japan
}

(Dated: August 21, 2018)

\begin{abstract}
Hydrodynamical calculations have been successful in describing global observables in ultrarelativistic heavy ion collisions, which aim to observe the production of the quark-gluon plasma. On the other hand, recently, a lot of evidence that there exists a critical end point (CEP) in the QCD phase diagram has been accumulating. Nevertheless, so far, no equation of state with the CEP has been employed in hydrodynamical calculations. In this paper, we construct the equation of state with the CEP on the basis of the universality hypothesis and show that the CEP acts as an attractor of isentropic trajectories. We also consider the time evolution in the case with the CEP and discuss how the CEP affects the final state observables, such as the correlation length, fluctuation, chemical freezeout, kinetic freezeout, and so on. Finally, we argue that the anomalously low kinetic freezeout temperature at the BNL Relativistic Heavy Ion Collider suggests the possibility of the existence of the CEP.
\end{abstract}

\section{INTRODUCTION}

The structure of the QCD phase diagram is one of the most interesting and important topics in nuclear and particle physics, and a lot of intensive studies have been carried out from both theoretical side and experimental side 1]. Recent theoretical studies have revealed the rich structure in the QCD phase diagram at finite density, which consists of not only the quark-gluon plasma (QGP) phase and hadron phase but also the two flavor color superconductor phase and color-flavor locked phase 2]. Furthermore, in the finite temperature direction, recent lattice calculations suggest the existence of mesonic bound states even above the deconfinement phase transition temperature [3, 4, 5, 6, 7, 8].

In this paper, we focus on the critical end point (CEP) in the QCD phase diagram, which is the terminating point of the first order phase transition ${ }^{1}$. In particular, we will consider its effects on the observables in ultrarelativistic heavy ion collisions in detail. Several effective theory analyses have predicted the existence of the CEP in the QCD phase diagram 10, 11, 12]. Since Fodor and Katz presented a QCD phase diagram with the CEP for the first time with lattice calculation [13], a lot of remarkable progress in finite temperature and density lattice calculation has been made [14, 15]. However, unfortunately, it will need more time before we reach the conclusive result on the existence of the CEP, its precise position, and so on, because finite density lattice QCD still has difficulties in performing accurate calculation on large lattices and at large chemical potential. Here, the

1 The terminology 'critical end point' is sometimes used for a different meaning [9]. However, it is rather customary in hadron physics to use it for the meaning we employ here. Thus, we use 'critical end point' instead of 'critical point' in this paper. We thank M. Stephanov for informing us of Ref. 9]. important point is that the existence of the CEP was shown semi-quantitatively by non-perturbative QCD calculation. The precise determination of the location of the CEP in the QCD phase diagram is a difficult problem. The locations of the CEP in different studies are, in fact, scattered over the $T$ (temperature) $-\mu_{B}$ (baryon chemical potential) plane [12]. Therefore, analyses from the experimental side are also indispensable in order to understand the properties of the QCD critical end point.

A promising way to investigate the existence and location of the QCD critical end point is to carry out experiments at various collision energies and compare their results. However, we have to find the observables which are suitable for detecting the CEP [16, 17, 18, 19, 20] for that purpose. In this paper, we study the CEP from the point of view of phenomenological analyses which can be compared with experimental results easily and discuss the indication of the existence of the CEP in experimental observables.

We divide the discussion into three steps. First, we construct equations of state that include the CEP. This step is necessary in order to consider the hydrodynamical evolution of the system. The guiding principle here is the theory of critical phenomena, in particular, the universality hypothesis. Second, we consider the deviation from the thermal equilibrium near the CEP. This is important because i) the typical time scale is elongated near the CEP, and ii) the system produced in ultrarelativistic heavy ion collisions cannot stay near the CEP due to the strong expansion. Finally, we discuss the possibility to observe the consequences of the CEP experimentally by using the preceding results.

The structure of this paper is as follows. In Section II we argue the general feature of the equation of state with the CEP on the basis of the universality hypothesis, the focusing effect in isentropic trajectories in the $T-\mu_{B}$ plane, and hydrodynamical expansion near the CEP. In Section III we investigate the growth and decrease of the 
correlation length near the CEP from the point of view of slowing-out-of-equilibrium. In Section IV we discuss the consequences of the CEP in ultrarelativistic heavy ion collision experiments. Section V is devoted to summary.

\section{EQUATION OF STATE WITH THE CRITICAL END POINT}

In this section, we construct realistic equations of state including the CEP. The equation of state with the CEP consists of two parts, the singular part and non-singular part. We assume that the CEP in QCD belongs to the same universality class as that in the three dimensional Ising model on the basis of the universality hypothesis. After mapping the variables and the equation of state near the CEP in the three dimensional Ising model onto those in $\mathrm{QCD}$, we match the singular entropy density near the CEP with the non-singular QGP and hadron phase entropy densities which are known away from the CEP. From this procedure we determine the behavior of the entropy density which includes both the singular part and non-singular part in a large region in the $T$ - $\mu_{B}$ plane. Finally, from the entropy density, we extract the behavior of other thermodynamical quantities such as the baryon number density, pressure, and energy density, and show that in the $T-\mu_{B}$ plane the CEP acts as an attractor of isentropic trajectories, $n_{B} / s=$ const., with $n_{B}$ and $s$ being the baryon number density and entropy density, respectively.

\section{A. Singular Part of Equation of State}

In the three dimensional Ising model, the magnetization $M$ (the order parameter) is a function of the reduced temperature $r=\left(T-T_{c}\right) / T_{c}$ and the external magnetic field $h$ with $T_{c}$ being the critical temperature. The CEP is located at the origin $(r, h)=(0,0)$. At $r<0$ the order of the phase transition is first and at $r>0$ it is crossover. A useful form of the equation of state of the three-dimensional Ising model is given through the parametric representation by the two variables $R$ and $\theta$,

$$
\left\{\begin{aligned}
M & =M_{0} R^{\beta} \theta, \\
h & =h_{0} R^{\beta \delta} \tilde{h}(\theta) \\
& =h_{0} R^{\beta \delta}\left(\theta-0.76201 \theta^{3}+0.00804 \theta^{5}\right), \\
r & =R\left(1-\theta^{2}\right) \quad(R \geq 0,-1.154 \leq \theta \leq 1.154),
\end{aligned}\right.
$$

where $M_{0}$ and $h_{0}$ are normalization constants and critical exponents $\beta$ and $\delta$ are 0.326 and 4.80, respectively 21]. We set the normalization constants $M_{0}$ and $h_{0}$ by imposing $M(r=-1, h=+0)=1$ and $M(r=0, h=1)=1$, which assures that the critical magnetization behaves as $M(r=0, h) \propto \operatorname{sgn}(h)|h|^{1 / \delta}$ and $M(r, h=+0) \propto|r|^{\beta}$ $(r<0)$ around the origin.

In order to determine the singular part of the entropy density, we start from the Gibbs free energy density
$G(h, r)$,

$$
G(h, r)=F(M, r)-M h,
$$

where $F(M, r)$ is the free energy density. Setting the free energy density,

$$
F(M, r)=h_{0} M_{0} r^{2-\alpha} g(\theta),
$$

together with the relation $h=(\partial F / \partial M)_{r}$, we obtain the differential equation for $g(\theta)$,

$$
\tilde{h}(\theta)\left(1-\theta^{2}+2 \beta \theta^{2}\right)=2(2-\alpha) \theta g(\theta)+\left(1-\theta^{2}\right) g^{\prime}(\theta),
$$

where a critical exponent $\alpha$ is 0.11 21]. Substituting $\tilde{h}(\theta)$ into Eq. (4), we obtain

$$
g(\theta)=g(1)+c_{1}\left(1-\theta^{2}\right)+c_{2}\left(1-\theta^{2}\right)^{2}+c_{3}\left(1-\theta^{2}\right)^{3},
$$

where $g(1)$ is determined by Eqs. (11) and (4),

$$
g(1)=\frac{\beta}{2-\alpha} \tilde{h}(1)
$$

and the coefficients $c_{1}, c_{2}$, and $c_{3}$ are given by

$$
\begin{aligned}
& c_{1}=-\frac{1}{2} \frac{1}{\alpha-1}\{(1-2 \beta)(1+a+b)-2 \beta(a+2 b)\}, \\
& c_{2}=-\frac{1}{2 \alpha}\{2 \beta b-(1-2 \beta)(a+2 b)\}, \\
& c_{3}=-\frac{1}{2(\alpha+1)} b(1-2 \beta),
\end{aligned}
$$

where $a=-0.76201$ and $b=0.00804$ are the coefficients of the $\theta^{3}$ and $\theta^{5}$ terms in $\tilde{h}(\theta)$, respectively [21]. Actually, there is freedom to add the function $C\left(1-\theta^{2}\right)^{2-\alpha}$ to $g(\theta)$, with $C$ being a constant. However, we will not take into account this part with a free parameter, because, whereas the universality tells us about only the critical behavior near the phase transition, this part does not show a singular behavior.

Differentiating the Gibbs free energy by the temperature, we obtain the singular part of the entropy density $s_{c}$ near the QCD critical end point,

$$
\begin{aligned}
s_{c} & =-\left(\frac{\partial G}{\partial T}\right)_{\mu_{B}} \\
& =-\left(\frac{\partial G}{\partial h}\right)_{r} \frac{\partial h}{\partial T}-\left(\frac{\partial G}{\partial r}\right)_{h} \frac{\partial r}{\partial T},
\end{aligned}
$$

where $\left(\frac{\partial G}{\partial h}\right)_{r}$ and $\left(\frac{\partial G}{\partial r}\right)_{h}$ are, respectively, given by

$$
\begin{aligned}
\left(\frac{\partial G}{\partial h}\right)_{r} & =\left(\frac{\partial F(M, r)}{\partial h}\right)_{r}-\left(\frac{\partial M}{\partial h}\right)_{r} h-M \\
& =-M,
\end{aligned}
$$




$$
\begin{aligned}
\left(\frac{\partial G}{\partial r}\right)_{h} & =\left(\frac{\partial F(M, r)}{\partial r}\right)_{h}-\left(\frac{\partial M}{\partial r}\right)_{h} h \\
& =\frac{h_{0} M_{0} R^{1-\alpha}}{2 \beta \delta \theta \tilde{h}(\theta)+\left(1-\theta^{2}\right) \tilde{h}^{\prime}(\theta)} \times\left\{(2-\alpha) \tilde{h}^{\prime}(\theta) g(\theta)-\beta \theta \tilde{h^{\prime}}(\theta) \tilde{h}(\theta)-\beta \delta \tilde{h}(\theta) g^{\prime}(\theta)+\beta \delta \tilde{h}^{2}(\theta)\right\} .
\end{aligned}
$$

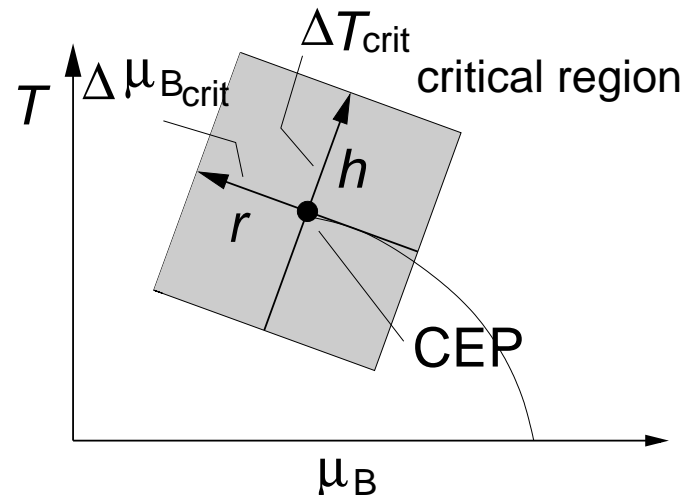

FIG. 1: Sketch of the $r-h$ axes (three dimensional Ising model) mapped onto the $T-\mu_{B}$ plane (QCD). The $r$ axis is tangential to the QCD phase boundary at the CEP. We set the $h$ direction perpendicular to the $r$ direction.

Note that $T$ in Eq. (8) is the temperature on the QCD side. In Eqs. (9) and (10), we use the differential equation Eq. (44) and the following relations,

$$
\begin{aligned}
\left(\frac{\partial R}{\partial h}\right)_{r} & =\frac{1}{h_{0} R^{\beta \delta-1}} \frac{2 \theta}{2 \beta \delta \theta \tilde{h}(\theta)+\left(1-\theta^{2}\right) \tilde{h}^{\prime}(\theta)} \\
\left(\frac{\partial \theta}{\partial h}\right)_{r} & =\frac{1}{h_{0} R^{\beta \delta}} \frac{1-\theta^{2}}{2 \beta \delta \theta \tilde{h}(\theta)+\left(1-\theta^{2}\right) \tilde{h}^{\prime}(\theta)} \\
\left(\frac{\partial R}{\partial r}\right)_{h} & =\frac{\tilde{h^{\prime}}(\theta)}{2 \beta \delta \theta \tilde{h}(\theta)+\left(1-\theta^{2}\right) \tilde{h}^{\prime}(\theta)} \\
\left(\frac{\partial \theta}{\partial r}\right)_{h} & =-\frac{\beta \delta}{R} \frac{\tilde{h}(\theta)}{2 \beta \delta \theta \tilde{h}(\theta)+\left(1-\theta^{2}\right) \tilde{h}^{\prime}(\theta)}
\end{aligned}
$$

Now we map the $r$ - $h$ plane in the three dimensional Ising model onto the $T-\mu_{B}$ plane in QCD in order to determine $\partial h / \partial T$ and $\partial r / \partial T$ in Eq. (8). The CEP in the three dimensional Ising Model, which is the origin in the $r$ - $h$ plane, is mapped to the CEP in QCD, $\left(T, \mu_{B}\right)=$ $\left(T_{E}, \mu_{B E}\right)$. The $r$ axis is tangential to the first order phase transition line at the CEP 22]. However, there is no general rule about how the $h$ axis is mapped in the $T-\mu_{B}$ plane. Here, for simplicity, we set the $h$ axis perpendicular to the $r$ axis. In Fig. 1 at $r<0$ the order of the phase transition is first and at $r>0$ it is crossover.

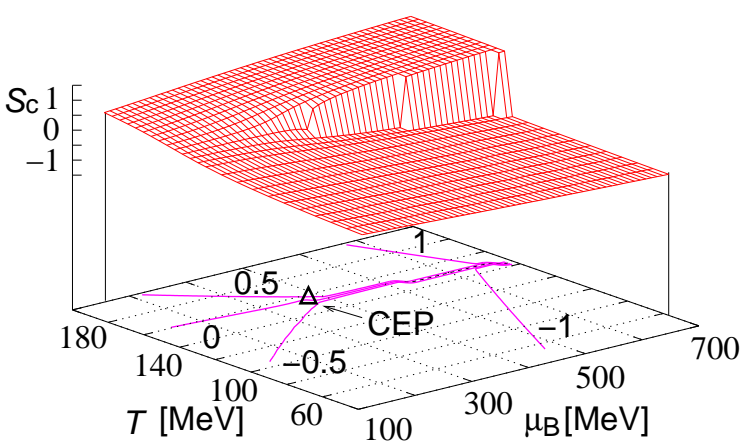

FIG. 2: Dimensionless variable $S_{c}\left(T, \mu_{B}\right)$ as a function of $T$ and $\mu_{B}$. The $\mathrm{CEP}$ is located at $\left(T, \mu_{B}\right)=$ (154.7 MeV,367.8 MeV) (the triangle in the contour plot).

\section{B. Thermodynamical Quantities}

For quantitative construction of equations of state with the CEP, we fix the relation between the scales in $(r, h)$ and $\left(T, \mu_{B}\right)$ variables, which provides the size of the critical region around the CEP in the $T-\mu_{B}$ plane, as follows: $\Delta r=1$ ( $r$ - $h$ plane $) \leftrightarrow \Delta \mu_{B \text { crit }}\left(T-\mu_{B}\right.$ plane $)$ and $\Delta h=1$ ( $r$ - $h$ plane $) \leftrightarrow \Delta T_{\text {crit }}\left(T-\mu_{B}\right.$ plane) (Fig. 11). $\Delta \mu_{B \text { crit }}$ and $\Delta T_{\text {crit }}$ give, respectively, approximate extensions of the critical region in the $\mu_{B}$ and $T$ directions when the location of the CEP is close to the $T$ axis and, as a result, the $r$ axis is approximately parallel to the $\mu_{B}$ axis, as recent lattice calculations suggest [13, 14, 15]. In order to connect the equations of state in the singular region and the non-singular region smoothly, we define the dimensionless variable $S_{c}\left(T, \mu_{B}\right)$ for the singular part of the entropy density $s_{c}$, which has the dimension [energy $]^{-1}$,

$$
S_{c}\left(T, \mu_{B}\right)=A\left(\Delta T_{\text {crit }}, \Delta \mu_{B \text { crit }}\right) s_{c}\left(T, \mu_{B}\right),
$$

where $A\left(\Delta T_{\text {crit }}, \Delta \mu_{B \text { crit }}\right)=\sqrt{\Delta T_{\text {crit }}^{2}+\Delta \mu_{B \text { crit }}^{2}} \times D$ and $D$ is a dimensionless constant. The extension of the critical domain around the CEP is specified by the parameters $\Delta T_{\text {crit }}, \Delta \mu_{B \text { crit }}$, and $D$. In Fig. 2 an example of $S_{c}$ is shown as a function of $T$ and $\mu_{B}$. The CEP is located at $\left(T, \mu_{B}\right)=(157.4 \mathrm{MeV}, 367.8 \mathrm{MeV})$ (the triangle in the contour plot). We use the same parameters $\left(\Delta T_{\text {crit }}, \Delta \mu_{B \text { crit }}, D\right)$ as those in Fig. 目 (left). $S_{c}$ is obtained by solving numerically the implicit relations derived in the preceding subsection. It is clearly seen that the order of the phase transition changes from crossover to first order at the CEP. The contour lines, $S_{c}= \pm 0.5$, 
give a rough idea about the size of the phase transition region. At lower $\mu_{B}$ below the critical chemical potential, the phase transition is smeared and the effect of the phase transition is observed in a larger domain in the temperature direction. Using the dimensionless variable $S_{c}\left(T, \mu_{B}\right)$, we define the entropy density in the $T-\mu_{B}$ plane,

$$
s\left(T, \mu_{B}\right)=\frac{1}{2}\left(1-\tanh \left[S_{c}\left(T, \mu_{B}\right)\right]\right) s_{\mathrm{H}}\left(T, \mu_{B}\right)+\frac{1}{2}\left(1+\tanh \left[S_{c}\left(T, \mu_{B}\right)\right]\right) s_{\mathrm{Q}}\left(T, \mu_{B}\right),
$$

where $s_{\mathrm{H}}$ and $s_{\mathrm{Q}}$ are the entropy densities in the hadron phase and QGP phase away from the CEP, respectively. This entropy density includes both singular and nonsingular contributions, and more importantly, gives the correct critical exponents near the QCD critical end point. $s_{\mathrm{H}}$ is calculated from the equation of state of the hadron phase in the excluded volume approximation 23],

$$
\begin{aligned}
P\left(T,\left\{\mu_{B i}\right\}\right) & =\sum_{i} P_{i}^{\text {ideal }}\left(T, \mu_{B i}-V_{0} P\left(T,\left\{\mu_{B i}\right\}\right)\right) \\
& =\sum_{i} P^{\text {ideal }}\left(T, \tilde{\mu}_{B i}\right),
\end{aligned}
$$

where $P$ is the pressure, $P_{i}^{\text {ideal }}$ is the pressure of the ideal gas of particle $i, \mu_{B i}$ is the baryon chemical potential of particle $i$, and $V_{0}$ is the hadron volume common to all hadron species. Non-strange resonances with mass up to $2 \mathrm{GeV}$ are included in the sum and the radius of hadrons is fixed at $0.7 \mathrm{fm}$. We obtain $s_{\mathrm{Q}}$ from the equation of state of the QGP phase in the Bag model,

$$
\begin{aligned}
P\left(T, \mu_{B}\right)= & \frac{\left(32+21 N_{f}\right) \pi^{2}}{180} T^{4}+\frac{N_{f}}{2}\left(\frac{\mu_{B}}{3}\right)^{2} T^{2} \\
& +\frac{N_{f}}{4 \pi^{2}}\left(\frac{\mu_{B}}{3}\right)^{4}-B
\end{aligned}
$$

where the number of the flavors $N_{f}$ is 2 and the bag constant $B$ is $(220 \mathrm{MeV})^{4}$. In Eq. (13) the relative strength of the singularity also depends on the distance between the QCD critical end point, where $S_{c}=0$, and the $s_{\mathrm{Q}}=s_{\mathrm{H}}$ line. If the CEP is located near the $s_{\mathrm{Q}}=s_{\mathrm{H}}$ line, less singularity is realized. However, in the following calculations, we assume that the CEP is on the phase transition line in the bag plus excluded volume model, where the phase transition is always of strong first order as shown later. Thus, $s_{\mathrm{Q}}$ is substantially larger than $s_{\mathrm{H}}$ at the CEP and the singularity is not suppressed.

Once we construct the entropy density, we can calculate the other thermodynamical quantities such as the baryon number density, pressure, and energy density. The baryon number density $n_{B}$ is given by

$$
\begin{aligned}
n_{B}\left(T, \mu_{B}\right) & =\frac{\partial P}{\partial \mu_{B}} \\
& =\int_{0}^{T} \frac{\partial s\left(T^{\prime}, \mu_{B}\right)}{\partial \mu_{B}} d T^{\prime}+n_{B}\left(0, \mu_{B}\right) .(16)
\end{aligned}
$$

In the first order phase transition region, in order to take into account the discontinuity in the entropy density and baryon number density on the phase boundary, it is necessary to add the following term to Eq. (16) for $T>T_{c}\left(\mu_{B}\right)$,

$$
\left|\frac{\partial T_{c}\left(\mu_{B}\right)}{\partial \mu_{B}}\right|\left(s\left(T_{c}\left(\mu_{B}\right)+0, \mu_{B}\right)-s\left(T_{c}\left(\mu_{B}\right)-0, \mu_{B}\right)\right),
$$

where $T_{c}\left(\mu_{B}\right)$ is the temperature of the first order phase transition at $\mu_{B}$. The pressure $P$ is obtained by integrating the entropy density with regard to the temperature,

$$
P\left(T, \mu_{B}\right)=\int_{0}^{T} s\left(T^{\prime}, \mu_{B}\right) d T^{\prime}+P\left(0, \mu_{B}\right) .
$$

Finally, from the thermodynamical relation the energy density $E$ is obtained as

$$
E=T s-P-\mu_{B} n_{B} .
$$

Here we make a comment on the choice of the parameters $\Delta T_{\text {crit }}, \Delta \mu_{B \text { crit }}$, and $D$. The linear mapping from $(r, h)$ to $\left(T, \mu_{B}\right)$, strictly speaking, holds only in the proximity of the CEP, where the thermodynamical quantities comply with the universality. We use the linear mapping throughout the critical region. In order to avoid artifacts due to this simplification, $\Delta T_{\text {crit }}, \Delta \mu_{B \text { crit }}$, and $D$ need to be appropriately chosen so that thermodynamical constraints are not violated. In particular, the following thermodynamical inequalities [22, 24, 25] need to be observed,

$$
\begin{aligned}
& \left(\frac{\partial S}{\partial T}\right)_{V, N_{B}}>0, \\
& \left(\frac{\partial P}{\partial V}\right)_{T, N_{B}}>0, \\
& \left(\frac{\partial \mu_{B}}{\partial N_{B}}\right)_{T, V}>0,
\end{aligned}
$$

where $S$ is the entropy, $N_{B}$ is the baryon number, and $V$ is the volume of the system.

Figure 3 indicates the entropy density and the baryon number density as a function of $T$ and $\mu_{B}$. We can see that in the high chemical potential region the order of 
the phase transition is first and that in the low chemical potential region a smooth phase transition occurs, which reflects the existence of the CEP. In this calculation the CEP is located at $\left(T, \mu_{B}\right)=(154.7 \mathrm{MeV}, 367.8 \mathrm{MeV})$. Since the universality does not provide the information on the location of the CEP, it is treated as a parameter throughout this paper as well as the values of $\Delta T_{\text {crit }}$, $\Delta \mu_{B \text { crit }}$, and $D$. Note, however, that the local singular behavior of thermodynamical quantities around the CEP is fixed by the universality hypothesis and that accordingly the local features such as the focusing, which we discuss below, are not affected by this ambiguity.
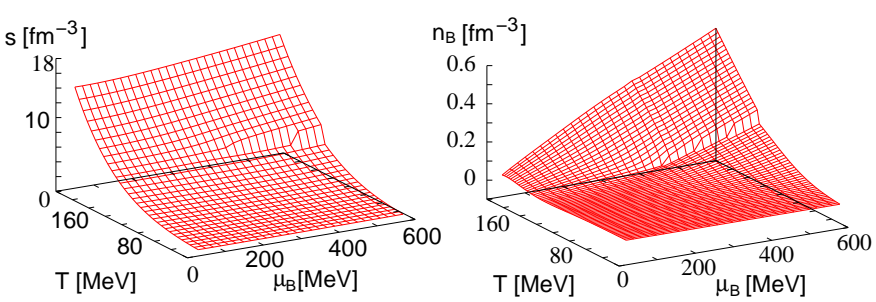

FIG. 3: Entropy density (left) and the baryon number density (right) as a function of $T$ and $\mu_{B} . \quad\left(T_{\mathrm{E}}, \mu_{B \mathrm{E}}\right)=$ $(154.7 \mathrm{MeV}, 367.8 \mathrm{MeV})$. The values of $\left(\Delta T_{\text {crit }}, \Delta \mu_{\text {Bcrit }}, D\right)$ are the same as in Fig. 4 (left).

First we investigate the behavior of the isentropic trajectories, i.e., contour lines of $n_{B} / s$. When entropy production can be ignored, the entropy and baryon number are conserved in each volume element and, therefore, the temperature and chemical potential in a given volume element change along the contour lines specified by the initial condition.

Figure 4 shows the isentropic trajectories in the $T-\mu_{B}$ plane. The values of $\left(\Delta T_{\text {crit }}, \Delta \mu_{B \text { crit }}, D\right)$ for the Fig. 4 (left) and Fig. 4 (right) are (100 MeV, $200 \mathrm{MeV}, 0.15$ ) and $(100 \mathrm{MeV}, 200 \mathrm{MeV}, 0.4)$, respectively. In both cases, the trajectories are focused to the CEP. This 'focusing' effect is more clearly observed in the case of $\left(T_{\mathrm{E}}, \mu_{B \mathrm{E}}\right)=(143.7 \mathrm{MeV}, 652 \mathrm{MeV})$ (Fig. 4 (right)). Thus, the CEP acts as an attractor of isentropic trajectories. Figure 5 shows isentropic trajectories in the bag plus excluded volume model, which is currently employed in most of hydrodynamical calculations 26]. The order of the phase transition is always first in this case. There is no focusing effect on the isentropic trajectories in this case. Instead, the trajectories are just shifted to the left on the phase transition line. This implies that the hydrodynamical evolution in the case with the CEP is very different from the one in the case with the equation of state given by the bag plus excluded volume model. Because of this attractor character of the CEP, it is not needed to fine-tune the collision energy to make the system pass near the CEP, which is pointed out in Ref. 16] for the first time. In other words, it is expected that the effect of the CEP, if any, changes only slowly as the collision energy is changed.
Two comments are in order here. First, as we explained, the universality does not tell us about the sizes of $\Delta T_{\text {crit }}, \Delta \mu_{B \text { crit }}, D$, and so forth. If $D$ is larger, the size of the critical region becomes smaller and the behavior of the isentropic trajectories approaches that in Fig. 5] which can be seen from Eqs. (12) and (13). Namely in the case of larger $D$, the contour lines of $S_{c}$ (for example, $S_{c}=-1,-0.5,0.5$ and 1 in Fig. 2) are closer to the $S_{c}=0$ line, i.e., the tangential line to the QCD phase boundary at the CEP. Then, in the limit of $D \rightarrow \infty$, all contour lines shrink to one line, which is identical to the QCD phase boundary as long as the curvature of the phase boundary can be ignored. This can be seen from Eq. (13). Therefore, the bag plus excluded volume model can be considered as an extreme case of the equation of state with the CEP. Second, the focusing on the right hand side of the CEP was first discussed in Ref. [16]. Our numerical result indicates that the CEP attracts isentropic trajectories not only on the right hand side (first order side) of the CEP but also on the left hand side (crossover side) of the CEP. Thus, if the critical region is large enough, the fine-tuning of the collision energy is not necessary to hit the vicinity of the CEP not only on the low energy side [16] but also on the high energy side.
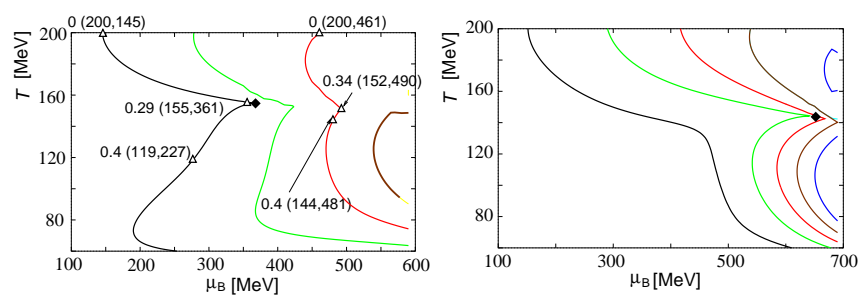

FIG. 4: Isentropic trajectories in the cases with the CEP. The $\mathrm{CEP}$ is located at $\left(T_{\mathrm{E}}, \mu_{B \mathrm{E}}\right)=(154.7 \mathrm{MeV}, 367.8 \mathrm{MeV})$ (left) and $\left(T_{\mathrm{E}}, \mu_{B \mathrm{E}}\right)=(143.7 \mathrm{MeV}, 652.0 \mathrm{MeV})$ (right). The values of $n_{B} / s$ on the trajectories are $0.01,0.02,0.03$, and 0.04 (left) and $0.01,0.02,0.03,0.04$, and 0.05 (right) from left to right. $L / L_{\text {total }}\left(T, \mu_{B}\right)$ is shown for some points on two trajectories in the left figure.

Next we argue the change of the square of the sound velocity $c_{s}^{2}$,

$$
c_{s}^{2}=\left(\frac{\partial P}{\partial E}\right)_{n_{B} / s},
$$

along the isentropic trajectories, which gives us the information on how the equation of state changes in the course of the hydrodynamical evolution. Figure 6] indicates the sound velocity as a function of $L / L_{\text {total }}$, where $L$ is the path length to a point along the isentropic trajectory with a given $n_{B} / s$ from a reference point on the same isentropic trajectory on the $T-\mu_{B}$ plane, and $L_{\text {total }}$ is the one to another reference point along the trajectory. For example, on the insentropic trajectory $n_{B} / s=0.01$ in Fig. 4 (left), $L / L_{\text {total }}=0$, which is the starting point of $n_{B} / s$ trajectory, corresponds to the point $\left(T, \mu_{B}\right)=$ 


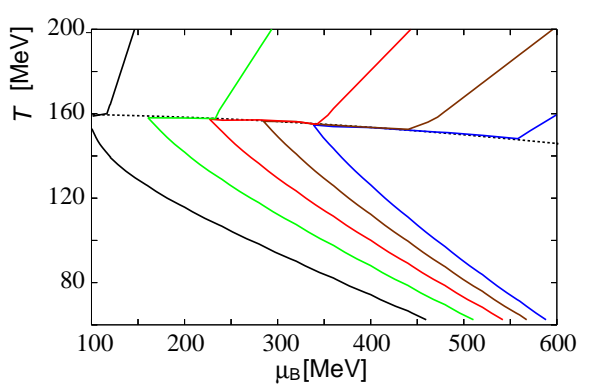

FIG. 5: Isentropic trajectories (solid lines) in the bag plus excluded volume model. The values of $n_{B} / s$ on the trajectories are $0.01,0.02,0.03,0.04$, and 0.05 from left to right. The dashed line stands for the phase boundary. The order of the phase transition is always first in this case.

$(200 \mathrm{MeV}, 145 \mathrm{MeV})$ and $L / L_{\text {total }}=0.29$ corresponds to the point $\left(T, \mu_{B}\right)=(155 \mathrm{MeV}, 361 \mathrm{MeV})$. At about $L / L_{\text {total }}=0.25 \sim 0.35$ the hadronization process occurs and at about $L / L_{\text {total }}=0.35 \sim 0.5$ kinetic freezeout takes place. We can see clear difference between the behavior of the sound velocities on $n_{B} / s=0.01$ and 0.03 trajectories. In the case of $n_{B} / s=0.01$, the sound velocity changes smoothly along the trajectory, which reflects the occurrence of a smooth phase transition at this $n_{B} / s$ value, and it takes its minimum at $L / L_{\text {total }}=0.29$. On the other hand, the sound velocity at $n_{B} / s=0.03$ changes suddenly at $L / L_{\text {total }}=0.34$, due to the first order phase transition. The hydrodynamical expansion along various $n_{B} / s$ paths differs in how the effect of the phase transition appears because of the existence of the CEP. In a real collision system, $n_{B} / s$ changes from position to position. As a result, the time evolution of such a system is described as a superposition of trajectories with different $n_{B} / s$ 's. Thus, the system may not expand uniformly and this effect may appear in physical observables related to expansion such as the collective flow and Hanbury Brown-Twiss (HBT) radii if the collision parameters are appropriate, i.e., isentropic trajectories in the system pass through and/or near the CEP.

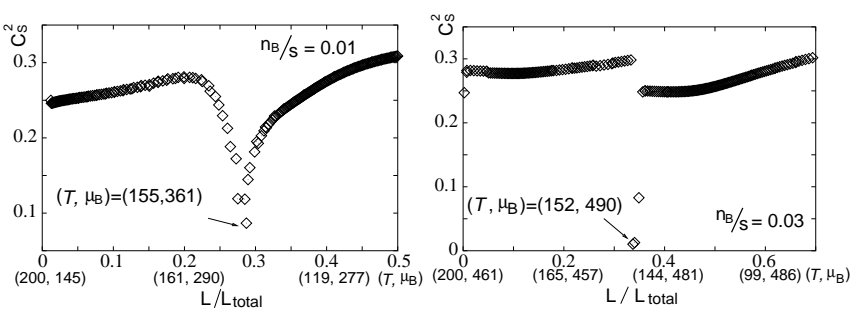

FIG. 6: Square of the sound velocity $c_{s}^{2}$ as a function of $L / L_{\text {total }}$ along $n_{B} / s=0.01$ (left) and $n_{B} / s=0.03$ (right) isentropic trajectories. The CEP is located at $\left(T_{E}, \mu_{B E}\right)=$ $(154.7 \mathrm{MeV}, 367.8 \mathrm{MeV})$ in both cases. The parameters are the same as in Figs. 2, 3, and 4 (left).

\section{SLOWING OUT OF EQUILIBRIUM}

The equilibrium correlation length and fluctuation become large near the CEP and they diverge at the CEP. At the same time, the typical time scale becomes long near the CEP, which makes the system take long time to reach the thermal equilibrium near the CEP. Therefore, in the time evolution in ultrarelativistic heavy ion collisions, near the CEP slowing-out-of-equilibrium occurs and nonequilibrium dynamics has to be taken into account. We assume that the thermal equilibrium is established soon after collisions and that the quark-gluon plasma follows Bjorken's scaling solution [27] for hydrodynamical evolution. The initial temperature and proper time are set to $200 \mathrm{MeV}$ and $1 \mathrm{fm} / c$, respectively ${ }^{2}$. Before discussing the correlation length in out-of-equilibrium time evolution, we calculate the equilibrium correlation length near the CEP in the $r$ - $h$ plane. Using Widom's scaling law [28], the equilibrium correlation length $\xi_{\text {eq }}$ is given by

$$
\xi_{\text {eq }}^{2}(r, M)=a^{2} M^{-2 \nu / \beta} g\left(\frac{|r|}{|M|^{1 / \beta}}\right),
$$

where $\nu=0.63$ is a critical exponent of the three dimensional Ising model 21]. $a$ is a constant with the dimension of length and fixed to $1 \mathrm{fm}$. There is not so large ambiguity in the determination of $a$, since $\xi_{\text {eq }}$ is of the order of $1 \mathrm{fm}$ at $T \sim 200 \mathrm{MeV}$ The function $g(x)$ is given by the $\epsilon$ expansion to order $\epsilon^{2}$ [28],

$$
\begin{aligned}
g(x)= & g_{\epsilon}(x) \\
= & 6^{-2 \nu} z\left\{1-\frac{\epsilon}{36}[(5+6 \ln 3) z-6(1+z) \ln z]\right. \\
& +\epsilon^{2}\left[\frac{1+2 z^{2}}{72} \ln ^{2} z+\left(\frac{z}{18}\left(z-\frac{1}{2}\right)(1-\ln 3)\right.\right. \\
& \left.-\frac{1}{216}\left(16 z^{2}-\frac{47}{3} z-\frac{56}{3}\right)\right) \ln z \\
& +\frac{1}{216}\left(\frac{101}{6}+\frac{2}{3} I+6 \ln ^{2} 3+4 \ln 3-10\right) z^{2} \\
& \left.\left.-\frac{1}{216}\left(6 \ln ^{2} 3+\frac{44}{3} \ln 3+\frac{137}{9}+\frac{8}{3} I\right) z\right]\right\},
\end{aligned}
$$

where $z \equiv \frac{2}{1+x / 3}, I \equiv \int_{0}^{1} \frac{\ln [x(1-x)]}{1-x(1-x)} d x \sim-2.344$, and $\epsilon=4-d$ with $d$ being the dimension of the space, 3 . At large $x$, i.e., around the crossover and CEP, $g_{\epsilon}(x)$ cannot be used and the asymptotic form given in Ref. [29],

$$
g(x)=g_{\text {large }}(x)=\left(\frac{1}{3+x}\right)^{2 \nu},
$$

${ }^{2}$ In Bjorken's one-dimensional hydrodynamical evolution, the non-equilibrium correlation length is not sensitive to the choice of the initial conditions. Instead, as we discuss later, the reduction of the time scale due to the transverse expansion is expected to have more effect on it. 
should be used. We smoothly connect $g_{\epsilon}(x)$ and $g_{\text {large }}(x)$ around $x=5$, i.e., in most part of the critical region $g(x)$ is given by Eq. (23).

Figure 7 shows the correlation length in equilibrium and the isentropic trajectories at some $n_{B} / s$ 's in the $r$ - $h$ plane. In Fig. 7 the CEP is located at the origin, and the first order phase transition occurs at $r<0$ and the crossover phase transition takes place at $r>0$. The correlation length in equilibrium $\xi_{\text {eq }}$ is divergent at the origin and the region where $\xi_{\text {eq }}$ is large spreads out in the smooth phase transition region, $r>0$. In Fig. 7 solid lines stand for the isentropic trajectories in the case of $\left(T_{E}, \mu_{B E}\right)=(154.7 \mathrm{MeV}, 367.8 \mathrm{MeV})$ (left) and $\left(T_{E}, \mu_{B E}\right)=(143.7 \mathrm{MeV}, 652.0 \mathrm{MeV})$ (right). Note that this $r$ - $h$ plane is actually placed in the $T-\mu_{B}$ plane with the tilt given by the slope of the tangential line of the first order phase transition line at the CEP as shown in Fig. 11 The value of $n_{B} / s$ is determined not only by $r$ and $h$ but also by the non-critical component of the equation of state. This is the reason why the behavior of the trajectories relative to the CEP changes according to the location of the CEP in the $T-\mu_{B}$ plane. Thus, the strength of focusing effect of the CEP is determined also by the non-singular part. On the other hand, we use the universality hypothesis to calculate $\xi_{\text {eq }}$ and, as a result, it is a function of only $r$ and $h$. The trajectories are attracted to the CEP, which makes the length of each trajectory in the critical region, where $\xi_{\text {eq }}$ is large, long. This behavior is very different from the assumption in usual schematic analyses, for example, Fig. 1 in Ref. 29] by Berdnikov and Rajagopal. The maximum value of $\xi_{\text {eq }}$ depends on the value of $n_{B} / s$.
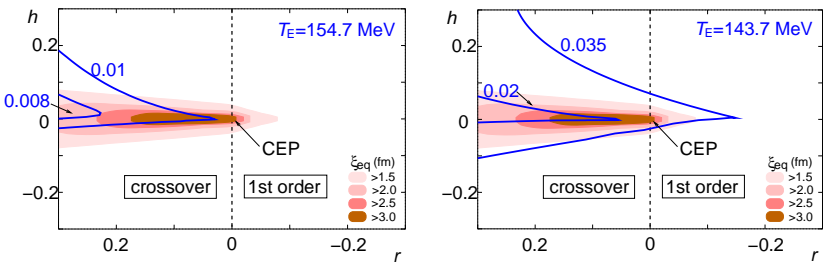

FIG. 7: Correlation length in equilibrium and the $n_{B} / s$ contour lines in the $r-h$ plane. The solid lines are trajectories with fixed $n_{B} / s$ values 0.008 and 0.01 in the case of $\left(T_{E}, \mu_{B E}\right)=(154.7 \mathrm{MeV}, 367.8 \mathrm{MeV})$ (left) and 0.02 and 0.035 in the case of $\left(T_{E}, \mu_{B E}\right)=(143.7 \mathrm{MeV}, 652.0 \mathrm{MeV})$ (right).

Next we calculate the non-equilibrium correlation length $\xi(\tau)$ as a function of the proper time $\tau$ by using the rate equation given in Ref. 29],

$$
\frac{d}{d \tau} m_{\sigma}(\tau)=-\Gamma\left[m_{\sigma}(\tau)\right]\left(m_{\sigma}(\tau)-\frac{1}{\xi_{\mathrm{eq}}(\tau)}\right)
$$

where $1 / m_{\sigma}(\tau)=\xi(\tau)$ and $\Gamma\left[m_{\sigma}(\tau)\right]$ is the parameter which represents the rate of slowing-out-of-equilibrium. From the theory of dynamical critical phenomena,
$\Gamma\left[m_{\sigma}(\tau)\right]$ is given as

$$
\Gamma\left[m_{\sigma}(\tau)\right]=\frac{A}{\xi_{0}}\left(m_{\sigma}(\tau) \xi_{0}\right)^{z},
$$

where we take $\xi_{0}=\xi_{\text {eq }}(T=200 \mathrm{MeV})$, and $z$ is a universal exponent of Model $\mathrm{H}$ in [30], i.e., $z \sim 3$ [29, 31]. $A$ is a dimensionless non-universal parameter and we use $A=1$ as in Ref. 29], though there is considerable uncertainty in the parameter $A$. However, fortunately, the relation between $\xi_{\text {eq }}$ and $\xi$ does not depend on the parameter choice of $A$ so much at the kinetic freezeout point. The non-equilibrium correlation length approaches the equilibrium correlation length as $A$ increases. At $A=100, \xi$ is almost equal to $\xi_{\text {eq }}$. On the other hand, at small $A$ the effect of slowing-out-of-equilibrium appears strongly. At the same time, however, the maximum value of $\xi$ becomes small as $A$ decreases. As a result, the absolute value of the difference between $\xi_{\text {eq }}$ and $\xi$ at the kinetic freezeout temperature remains almost unchanged for a wide range of $A$. Indeed, we have checked that the following discussion about non-equilibrium correlation length holds for $0.1 \lesssim A \lesssim 10$

Figure 8 shows the correlation length as a function of $L / L_{\text {total }}$. The dashed and solid lines stand for the correlation lengths in equilibrium at $n_{B} / s=0.008$ and $n_{B} / s=0.01$, respectively. The parameters are the same as for Fig. 4 (left). The thin and thick lines are $\xi_{\text {eq }}$ and $\xi$, respectively. The maximum value of $\xi_{\text {eq }}$ along the former trajectory is larger than that along the latter, because the former approaches the CEP more closely than the latter. The non-equilibrium correlation length $\xi$ is smaller than $\xi_{\text {eq }}$ at the beginning. Then, $\xi$ becomes larger than $\xi_{\text {eq }}$ later. These are both due to the critical slowing down around the CEP, as pointed out in Ref. 29]. However, the difference becomes small by the time the system gets to the kinetic freezeout point. If the transverse expansion is taken into account, the time scale in the hadron phase becomes much shorter, but $\xi_{\text {eq }}$ is already small in the hadron phase and the difference is expected to remain small. Figure 9 shows the cube of the correlation length as a function of the temperature for $n_{B} / s=0.008,0.01$, and 0.015. The parameters are the same as for Fig. 8 The fluctuation of the sigma field scales approximately as $\propto \xi^{3}$. The singular part of the fluctuation of intensive thermal quantities of pions near the CEP is proportional to $\xi^{2}[16]$. The general relation between $\xi$ and observed fluctuations still needs to be clarified. These relations, however, suggest that there is room for the observation of some enhancement in fluctuations when the system passes the vicinity of the CEP even if the enhancement of $\xi$ is small.

\section{CONSEQUENCES OF THE CRITICAL END POINT IN EXPERIMENTS}

Many experimental analyses have been carried out in search of the evidence of the CEP in the QCD phase 


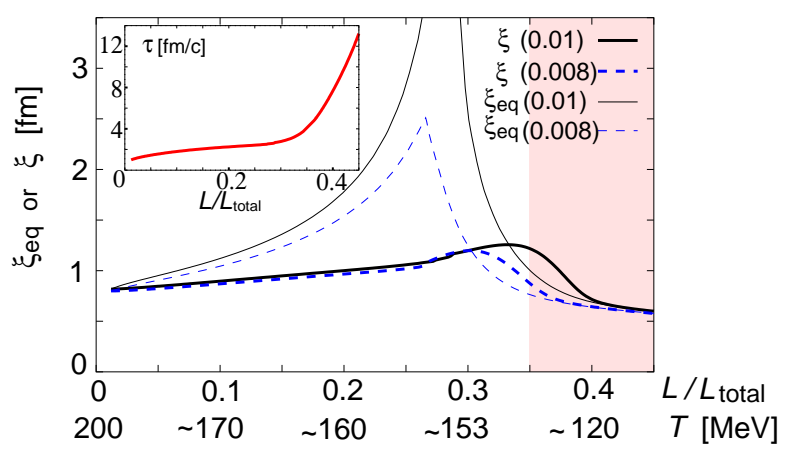

FIG. 8: Equilibrium correlation length $\xi_{\text {eq }}$ (thin lines) and non-equilibrium correlation length $\xi$ (thick lines) on the isentropic trajectories with $n_{B} / s=0.008$ and 0.01 , together with $\tau$ as functions of $L / L_{\text {total }}$ (inlet). $z=3$ was used in the calculation.

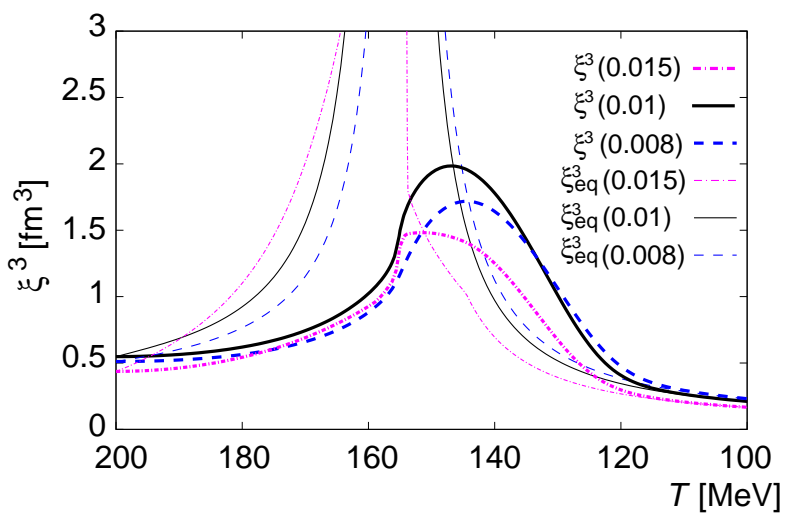

FIG. 9: Cube of the equilibrium correlation length (thin lines) as a function of the temperature and the cube of the non-equilibrium correlation length (thick lines) on the isentropic trajectories with $n_{B} / s=0.008,0.01$, and 0.015 . The parameters are the same as for Fig. 8 The isentropic lines with $n_{B} / s=0.008,0.01$, and 0.015 pass left of, almost through, and right of the CEP, respectively.

diagram. Since the correlation length and fluctuation diverge at the CEP in thermal equilibrium, it has been expected that some enhancement of the fluctuation, for instance, is observed if the collision energy of nuclei is properly adjusted so that the system goes right through the CEP. Generally, the higher the collision energy is, the smaller chemical potential region is explored in the $T-\mu_{B}$ plane. Thus, it has also been expected that the observables related to the critical behavior around the $\mathrm{CEP}$ such as fluctuations show non-monotonic behavior as a function of the collision energy. We discuss the observability of such behavior in ultrarelativistic heavy ion collisions on the basis of our findings in the previous sections.

It has been naively expected that the collision energy needs to be carefully adjusted so that the system goes right through the CEP and its existence can be confirmed. In section II, we have shown that the CEP acts

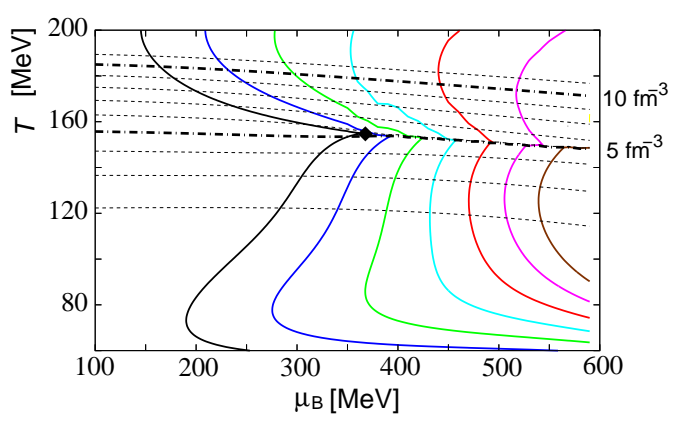

FIG. 10: Isentropic trajectories and entropy density contour lines in the case with the CEP. The parameters are the same as for Fig. 3 and the left figure of Fig. 4 The solid lines are isentropic trajectories $\left(n_{B} / s=0.01,0.015,0.02,0.025,0.03\right.$, 0.035 , and 0.04 from left to right) as in Fig. 4 The dashed lines are entropy density contour lines, which are shown at every $1 \mathrm{fm}^{-3}$.

as an attractor of the isentropic trajectories. Thus, if the size of the critical domain is large enough, as stressed in Ref. 16] for the right hand side of the CEP, it is not necessary to fine-tune the collision energy to make the system approach the CEP closely enough. However, such physical quantities that show the critical behavior near the CEP are hadronic observables, and are subject to final state interactions in the hadron phase. It has been often argued that if the system passes near the CEP, kinetic freezeout takes place near the CEP [16]. It is based on the expectation that, in such a case, the system stays long near the CEP, where the phase transition is second order, and that when the system starts to leave away from the CEP, the entropy density or particle density is already small enough so that kinetic freezeout takes place. To the contrary, Fig. 3 does not show a sharp drop of the entropy density near the CEP. To see this more clearly, we show the contour plot of the entropy density in Fig.10 for the same parameters as in Fig. 3. The entropy density gives a semi-quantitative measure of the whereabouts of kinetic freezeout. Alternatively, we could use the energy density as a measure as well. Figure 10 shows that the contour lines of the entropy density are not focused near the CEP in contrast to the isentropic trajectories. Thus, it is not likely that kinetic freezeout takes place near the CEP. The reason can be traced back to the fact that at the CEP the entropy density is continuous and bound, although its derivative along the $h$ axis diverges. If the system does not freeze out near the CEP, the dilution of the critical behaviors in the hadron phase needs to be carefully considered.

Empirically it has been known that chemical freezeout takes place just below the theoretically expected phase transition line. Since the isentropic trajectories are focused around the CEP, it would be tempting to expect that the chemical freezeout points are focused as the collision energy is varied. However, so far, only the free resonance gas model has been used in the analysis of 
the chemical freezeout point. If the critical region is so large that the focusing is realized in a large region, the effect of the interactions on hadrons can by no means be ignored. Then, the hadrons at chemical freezeout are at most quasi-particles which are quantum-mechanically different from the hadrons in the vacuum [32] and it is necessary to consider the time evolution of the quasiparticle states to relate the chemical freezeout points and the observed particle number ratios. This is beyond the scope of this paper and we would like to leave this as a future problem.

The entropy density in Fig. 3 shows the following feature. On the left hand side of the CEP, the entropy density changes smoothly, reflecting the fact that the phase transition here is crossover. The closer to the $T$ axis, the less rapid the change is. On the other hand, on the right hand side of the CEP, the phase transition is of first order, and the jump in the entropy density increases as the chemical potential increases. As a result, i) the separation of the contour lines of the entropy density and energy density gets larger as the distance from the CEP becomes larger on the left hand side of the CEP, ii) the contour lines below the crossover are flatter than those in the hadron phase in the case with a first order phase transition (See Figs. 3] and 10). This feature is not due to the universality. Instead, it is a general consequence of the existence of the CEP. In fact, as shown in Fig. 11] this feature is not observed in the case of the bag plus excluded volume model. Using this feature of the entropy density, we discuss the behavior of kinetic freezeout as a function of the collision energy. Kinetic freezeout takes place when the mean (elastic) collision rate and the expansion rate of the system become comparable. The expansion rate in central collisions at RHIC is known to be considerably larger than that at SPS 33]. The elastic collision rate can be estimated from the elastic cross sections for pions and baryons and their densities. Due to the chiral symmetry and the effect of the $\Delta$, the elastic $\pi-N$ cross section is considerably larger than that of $\pi-\pi$. On the other hand, in $\mathrm{Pb}+\mathrm{Pb}$ collisions at $158 \mathrm{AGeV}$ at SPS, $(p+\bar{p}) /\left(\pi^{+}+\pi^{-}\right) \sim 0.09$ 34, 35] and it is $\sim 0.09$ at RHIC [36]. Thus, at the same particle number density, the collision rate at SPS is almost the same as that at RHIC. From these considerations, it would be natural if the kinetic freezeout temperature in central collisions at RHIC is noticeably higher than that at SPS, while the kinetic freezeout temperature at RHIC is actually slightly lower than that at SPS 33]. Two comments are in order here. First, the kinetic freezeout we are considering here is that of pions and kaons. The analysis in Ref. 33. does not take into account the effect of resonance decays. When resonance decays are taken into account, the kinetic freezeout temperature becomes even lower 37]. Second, more precisely speaking, kinetic freezeout takes place when the mean collision rate and the product of the expansion rate and the typical scale of the system become comparable. However, according to the results of the HBT interferometry, the typical size at RHIC is

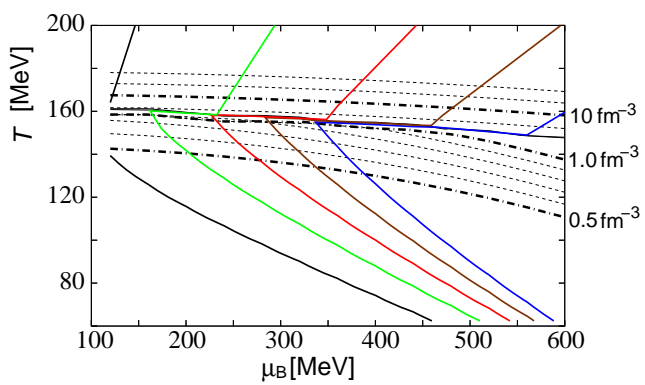

FIG. 11: Isentropic trajectories and entropy density contour lines in the case without the CEP. The parameters are the same as in Fig. 5 The solid lines are isentropic trajectories $\left(n_{B} / s=0.01,0.02,0.03,0.04\right.$, and 0.05 from left to right) as in Fig. 5] The dashed lines are the entropy density contour lines. The contour lines are shown at every $0.1(1) \mathrm{fm}^{-3}$ in the hadron (QGP) phases because the increase rate of the entropy density in the QGP phase is much larger than that in the hadron phase due to the strong first order phase transition.

not increased as the expansion rate compared to SPS.

This can be naturally understood if the separation of the entropy density ( particle density) or energy density contour lines is larger at kinetic freezeout at RHIC than at SPS. This implies that the CEP exists in the QCD phase diagram and, furthermore, that the RHIC isentropic trajectory passes on the left hand side of the CEP. To confirm this tendency, it is necessary to obtain the kinetic freezeout temperatures at several collision energies between the SPS and current RHIC energies.

In the previous section, we have shown that the difference between the thermal correlation length $\xi_{\text {eq }}$ and the actual correlation length $\xi$ is small at kinetic freezeout as well as $\xi$ itself. As explained in Ref. 29], precisely speaking, Eq. (26) is valid only near the CEP. Nevertheless, the physical reason why $\xi_{\text {eq }}$ and $\xi$ are so close to each other at kinetic freezeout, is general and holds also in this case; final state interactions tend to wash out nonequilibrium effects. Since the kinetic freezeout points at the Super Proton Synchrotron (SPS) at CERN and the Relativistic Heavy Ion Collider (RHIC) at Brookhaven National Laboratory are far below the phase transition line, the effect of final state interactions is expected to dominate.

Similar arguments hold also for fluctuations except for the cube effect we argued in the previous section. The event-by-event fluctuations of the mean transverse momentum in $\mathrm{Pb}+\mathrm{Au}$ collisions at 40, 80, and $158 \mathrm{AGeV} / c$ were measured by the CERES Collaboration [38]. They are slightly smaller than those at RHIC, and unusually large fluctuation or non-monotonic behavior which may suggest the existence of the CEP has not been observed. However, in order to understand experimental observations, we have to take account of the CEP character as an attractor of isentropic trajectories in the $T-\mu_{B}$ plane. Due to the focusing effect of the CEP, the isentropic trajectories of various initial collision energies are gathered 
to the CEP, if the critical region around the CEP is large enough. As a result, when each isentropic trajectory passes near the CEP, similar correlation lengths, fluctuations, and so on are induced, and they do not show strong non-monotonic behavior as a function of the collision energy. In other words, the absence of the non-monotonic behavior, which is shown by the CERES Collaboration, does not necessarily imply the non-existence of the CEP in the region probed by SPS and RHIC. More precise measurement and analyses which take account of the focusing and slowing-out-of-equilibrium may reveal the existence of the CEP.

Recently, the fluctuation of $\left(K^{+}+K^{-}\right) /\left(\pi^{+}+\pi^{-}\right)$ and $(p+\bar{p}) /\left(\pi^{+}+\pi^{-}\right)$was measured and it was found that the collision energy dependence of the fluctuation of $\left(K^{+}+K^{-}\right) /\left(\pi^{+}+\pi^{-}\right)$is not described by a cascade model 34]. While this result is interesting, we have to be careful in associating this result with the critical phenomena around the CEP. Since neither the numerator nor denominator of $\left(K^{+}+K^{-}\right) /\left(\pi^{+}+\pi^{-}\right)$is a conserved quantity in the strong interaction, substantial modification is expected for both in the hadron phase as we have demonstrated for the correlation length. Furthermore, as we discussed for the particle ratios, quasi-particle states near the CEP are quantum-mechanically different from the ones in the vacuum, i.e., observed pions, kaons, protons, and so on. To relate the fluctuation of the particle ratios to the critical phenomena, it is necessary to know not only the time evolution of the quasi-particle states but also the relation between the quasi-particle quantum states in medium and the particle states in the vacuum [32].

Up to now, we have been considering the isentropic trajectories in the $T-\mu_{B}$ plane. Actually, the viscosity is known to diverge at the CEP and the entropy is generated if the system passes near the CEP. However, the singularity in the viscosity is known to be very weak [31],

$$
\frac{\eta}{\eta_{0}}=\left(\frac{\xi_{\mathrm{eq}}}{\xi_{\mathrm{eq}, 0}}\right)^{\left(\frac{1}{19} \epsilon+O\left(\epsilon^{2}\right)\right)},
$$

where $\eta$ is the shear viscosity, $\eta_{0}$ is the shear viscosity at the point where the equilibrium correlation length is $\xi_{\mathrm{eq}, 0}$, and $\epsilon$ is $4-d$ as before. In Fig. 12] $\eta / \eta_{0}$ in the $r$ - $h$ plane is shown. The small correction to the critical exponent $1 / 19$ is set to 0 for simplicity. Because of the small critical exponent, the diverging feature of the viscosity is hardly seen. The effect of the entropy production around the CEP is thus expected to be small, and it will not show easily-recognizable non-monotonic changes in observables as the collision energy is changed.

\section{SUMMARY}

In this paper, we discussed the hydrodynamical expansion near the QCD critical end point and its consequences

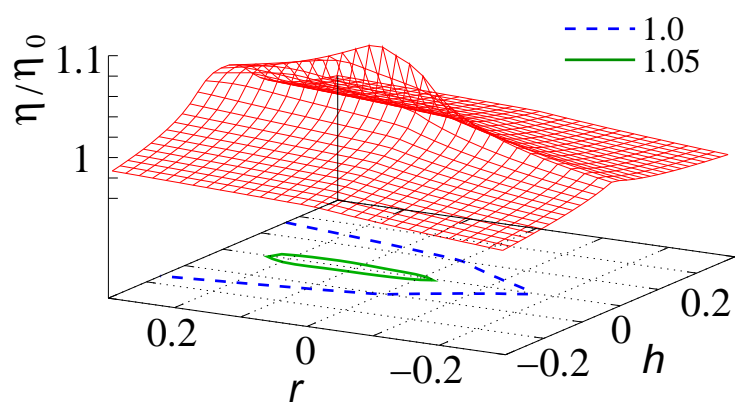

FIG. 12: Shear viscosity $\eta / \eta_{0}$ near the CEP. For the parameters used in the calculation, see the text.

in experimental observables. First we constructed realistic equations of state with the CEP on the basis of the universality hypothesis and discussed the hydrodynamical expansion near the CEP. The behavior of isentropic trajectories near the QCD critical end point is clearly different from that in the bag plus excluded volume model, which is usually used in hydrodynamical models. We found that the CEP acts as an attractor of isentropic trajectories, $n_{B} / s=$ const., in the $T-\mu_{B}$ plane, not only on the right hand side of the CEP but also on the left hand side of the CEP. Because of the focusing of the isentropic trajectories, the path of the system in the course of the time evolution in the $T-\mu_{B}$ plane is remarkably different from that in the usual bag plus excluded volume model. This will be reflected in the final state observables that are sensitive to the equation of state, such as the anisotropic flows (directed, elliptic, and higher order Fourier coefficients) 39, 40 and HBT radii. For this purpose, full 3-D hydrodynamical calculations [41, 42] with realistic equations of state are indispensable [43].

Next we argued the critical slowing down near the QCD critical end point. We compared the equilibrium and non-equilibrium correlation lengths along two isentropic trajectories which pass near the CEP. We found that the difference between them at kinetic freezeout is very small.

Furthermore, we considered the experimental observability of the consequences of the CEP. Fluctuations and viscosities diverge at the CEP, but fluctuation which is induced by the CEP will fade by kinetic freezeout for the same reason as for the correlation length. This explains the non-observation of sharp non-monotonic behaviors in fluctuations. However, the singular part of the fluctuations is approximately proportional to the cube of the correlation length and there is a possibility that more precise measurements in the future may reveal the existence of the CEP. The singularity in the viscosity is too weak to affect observables. Concerning the chemical freezeout process, we need to consider the effect of the interactions on hadrons for detailed discussion on particle number ratios, when it takes place near the CEP. We found that, at least now, the most promising way is to trace the behavior of the collision energy dependence of 
the kinetic freezeout temperature. The fact that the kinetic freezeout temperature at RHIC is lower than that at SPS suggests the existence of the CEP in the $T-\mu_{B}$ plane and that the RHIC path goes left of the CEP.

Although our discussion in this paper does not depend on the parameter choice in Eqs. (12) and (13) qualitatively, our assumption that the critical region near the CEP spreads out a large area may look too optimistic. However, according to recent lattice calculations at zero baryon chemical potential, the $\bar{\psi} \psi$ susceptibility shows a very broad peak as a function of the temperature. Furthermore, the width tends to even increase as the lattice spacing is decreased 44]. This clearly indicates that the free-gas description cannot be used at $100 \lesssim T \lesssim 250$ $\mathrm{MeV}$ at zero baryon chemical potential.

\section{Acknowledgments}

We thank B. Müller for illuminating discussions and E. Shuryak, M. Stephanov, and K. Rajagopal for comments. Also, we are grateful to O. Miyamura and S. Muroya for encouragement. C.N. was in part supported by DOE grants DE-FG02-96ER40945 and DE-FG02-03ER41239, and M.A. was in part supported by Grant-in-Aid by the Japanese Ministry of Education No. 14540255.

\section{APPENDIX A: STABILITY CONDITION}

In an isolated system, the entropy $S$ takes the maximum value in the stable equilibrium state and the fol- lowing relations [24, 25],

$$
\begin{array}{r}
\delta S=0, \\
\delta^{2} S<0,
\end{array}
$$

are satisfied. Here the independent variables are the energy $E$, volume $V$, and particle number $N$. Suppose that the total system whose volume is $V$ is composed of two parts with the volumes $V_{1}$ and $V_{2}$. Suppose that in $V_{1}\left(V_{2}\right)$ the variation of the energy, volume, and particle number are $\delta E_{1}\left(\delta E_{2}\right), \delta V_{1}\left(\delta V_{2}\right)$, and $\delta N_{1}\left(\delta N_{2}\right)$, respectively. These variations satisfy

$$
\begin{aligned}
\delta E_{1}+\delta E_{2} & =0 \\
\delta V_{1}+\delta V_{2} & =0 \\
\delta N_{1}+\delta N_{2} & =0 .
\end{aligned}
$$

By expanding $\delta S$ with regard to $E, V$, and $N$ in Eq. (A2) and substituting $\left(\frac{\partial S}{\partial E}\right)_{V, N}=\frac{1}{T},\left(\frac{\partial S}{\partial V}\right)_{E, N}=\frac{P}{T}$, $\left(\frac{\partial S}{\partial N}\right)_{E, V}=-\frac{\mu}{T}$, and Eq. (A3) into Eq. (A2), we obtain $T_{1}=T_{2}, P_{1}=P_{2}$, and $\mu_{1}=\mu_{2}$, i.e., both parts of the system have the same temperature, pressure, and chemical potential in equilibrium.

Next we evaluate the second variation $\delta^{2} S$ in Eq. (A2),

$$
\begin{aligned}
\delta^{2} S= & \left(\frac{\partial^{2} S_{1}}{\partial E_{1}^{2}}\right)_{V_{1}, N_{1}} \delta^{2} E_{1}+\left(\frac{\partial^{2} S_{1}}{\partial V_{1}^{2}}\right)_{E_{1}, N_{1}} \delta^{2} V_{1}+\left(\frac{\partial^{2} S_{1}}{\partial N_{1}^{2}}\right)_{E_{1}, V_{1}} \delta^{2} N_{1} \\
& +2\left(\frac{\delta^{2} S_{1}}{\delta E_{1} \delta V_{1}}\right)_{N_{1}} \delta E_{1} \cdot \delta V_{1}+2\left(\frac{\delta^{2} S_{1}}{\delta V_{1} \delta N_{1}}\right)_{E_{1}} \delta N_{1} \cdot \delta V_{1}+2\left(\frac{\delta^{2} S_{1}}{\delta E_{1} \delta N_{1}}\right)_{V_{1}} \delta E_{1} \cdot \delta N_{1} \\
& +(1 \rightarrow 2) .
\end{aligned}
$$

By calculating $\delta^{2} S$ using $T \delta S=\delta E+P \delta V-\mu \delta N$ and comparing it with (A4), we obtain

$$
T \delta^{2} S=-\delta T_{1} \delta S_{1}+\delta P_{1} \delta V_{1}-\delta \mu_{1} \delta N_{1}+(1 \rightarrow 2)
$$

By definition, 


$$
\begin{aligned}
& \delta S_{1}=\left(\frac{\partial S_{1}}{\partial T_{1}}\right)_{V_{1}, N_{1}} \delta T_{1}+\left(\frac{\partial S_{1}}{\partial V_{1}}\right)_{T_{1}, N_{1}} \delta V_{1}+\left(\frac{\partial S_{1}}{\partial N_{1}}\right)_{T_{1}, V_{1}} \delta N_{1}, \\
& \delta P_{1}=\left(\frac{\partial P_{1}}{\partial T_{1}}\right)_{V_{1}, N_{1}} \delta T_{1}+\left(\frac{\partial P_{1}}{\partial V_{1}}\right)_{T_{1}, N_{1}} \delta V_{1}+\left(\frac{\partial P_{1}}{\partial N_{1}}\right)_{T_{1}, V_{1}} \delta N_{1}, \\
& \delta \mu_{1}=\left(\frac{\partial \mu_{1}}{\partial T_{1}}\right)_{V_{1}, N_{1}} \delta T_{1}+\left(\frac{\partial \mu_{1}}{\partial V_{1}}\right)_{T_{1}, N_{1}} \delta V_{1}+\left(\frac{\partial \mu_{1}}{\partial N_{1}}\right)_{T_{1}, V_{1}} \delta N_{1} .
\end{aligned}
$$

Substituting Eq. A6 and the following relations, and using Eq. A3 , we obtain the quadratic form $\left(\frac{\partial S}{\partial T}\right)_{V, N}=\frac{1}{T} C_{v},\left(\frac{\partial S}{\partial V}\right)_{T, N}=\left(\frac{\partial P}{\partial T}\right)_{V, N},\left(\frac{\partial \mu}{\partial V}\right)_{T, N}=$ $-\left(\frac{\partial P}{\partial N}\right)_{T, V}$, and $\left(\frac{\partial \mu}{\partial T}\right)_{V, N}=-\left(\frac{\partial S}{\partial N}\right)_{T, V}$ into Eq. A5,

$$
T \delta^{2} S=-\frac{1}{T} C_{v}(\delta T)^{2}+\left(\frac{\partial P}{\partial V}\right)_{T, N}\left[\delta V+\frac{\left(\frac{\partial P}{\partial N}\right)_{T, V}}{\left(\frac{\partial P}{\partial V}\right)_{T, N}} \delta\right]^{2}-\left[\left(\frac{\partial \mu}{\partial N}\right)_{T, V}+\frac{\left(\frac{\partial P}{\partial N}\right)_{T, V}^{2}}{\left(\frac{\partial P}{\partial V}\right)_{T, N}}\right](\delta N)^{2},
$$

where we have suppressed the suffixes on the right hand side. Finally from Eqs. (A2) and (A7), we obtain the stability conditions,

$$
\begin{aligned}
& C_{v}>0 \\
&\left(\frac{\partial P}{\partial V}\right)_{T, N}<0, \\
&\left(\frac{\partial \mu}{\partial N}\right)_{T, V}>-\frac{\left(\frac{\partial P}{\partial N}\right)_{T, V}^{2}}{\left(\frac{\partial P}{\partial V}\right)_{T, N}}>0 .
\end{aligned}
$$

We note that A10 is equivalent to the following inequality [24],

$$
\left(\frac{\partial \mu}{\partial N}\right)_{P, T}>0
$$

[1] The proceedings of the Seventeenth International Conference on Ultra-Relativistic Nucleus-Nucleus Collisions (Quark Matter 2004), Oakland, U.S.A., 2004, J. Phys. G 30, S633 (2004).

[2] K. Rajagopal and F. Wilczek, Chapter 35 in the Festschrift in honor of B. L. Ioffe, At the Frontier of Particle Physics / Handbook of QCD, M. Shifman (ed.) (World Scientific), (hep-ph/0011333); D. Bailin and A. Love, Nucl. Phys. B 205, 119 (1982); M. Iwasaki and T. Iwado, Phys. Lett. B 350, 163 (1995); M. G. Alford, K. Rajagopal, and F. Wilczek, Phys. Lett. B 422, 247 (1998); R. Rapp, T. Schäfer, E. V. Shuryak, and M. Velkovsky, Phys. Rev. Lett. 81, 53 (1998); S. Muroya, A. Nakamura, C. Nonaka, and T. Takaishi, Prog. Theor. Phys. 110, 615 (2003).

[3] M. Asakakwa, T. Hatsuda, and Y. Nakahara, Nucl. Phys. Proc. Suppl. 119, 481 (2003).

[4] M. Asakawa and T. Hatsuda, Phys. Rev. Lett. 92, 012001 (2004).

[5] F. Karsch, E. Laermann, P. Petreczky, S. Stickan, and I. Wetzorke, Phys. Lett. B 530, 152 (2002).
[6] S. Datta, F. Karsch, P. Petreczky, and I. Wetzorke, Phys. Rev. D 69, 094507 (2004).

[7] H. Matsufuru, T. Umeda, and K. Nomura, hep-lat/0401010

[8] G. E. Brown, C.-H. Lee, M. Rho, and E. Shuryak, J. Phys. G 30, S1275 (2004).

[9] M. E. Fisher and M. C. Barbosa, Phys. Rev. B 43, 11177 (1991).

[10] M. Asakawa and K. Yazaki, Nucl. Phys. A 504, 668 (1989).

[11] M. A. Halasz, A. D. Jackson, R. E. Shrock, M. A. Stephanov, and J. J. M. Verbaarschot, Phys. Rev. D 58, 096007 (1998).

[12] M. Stephanov, Prog. Theor. Phys. Suppl. 153, 139 (2004).

[13] Z. Fodor and S. D. Katz, JHEP 0203, 014 (2002); JHEP 0404, 050 (2004).

[14] C. R. Allton, S. Ejiri, S. J. Hands, O. Kaczmarek, F. Karsch, E. Laermann, Ch. Schmidt, and L. Scorzato, Phys. Rev. D 66, 074507 (2002).

[15] Ph. de Forcrand and O. Philipsen, Nucl. Phys. B 673, 
170 (2003); hep-lat/0309109

[16] M. A. Stephanov, K. Rajagopal, and E. V. Shuryak, Phys. Rev. Lett. 81, 4816 (1998); M. A. Stephanov, K. Rajagopal, and E. V. Shuryak, Phys. Rev. D 60, 114028 (1999).

[17] Y. Hatta and T. Ikeda, Phys. Rev. D 67, 014028 (2003).

[18] K. Fukushima, Phys. Rev. C 67, 025203 (2003).

[19] Y. Hatta and M. A. Stephanov, Phys. Rev. Lett. 91, 102003 (2003).

[20] O. Scavenius, A. Mocsy, I. N. Mishustin, and D. H. Rischke, Phys. Rev. C 64, 045202 (2001).

[21] R. Guida and J. Zinn-Justin, Nucl. Phys. B 489, 626 (1997).

[22] W. Gebhardt and U. Krey, Phasenübergänge und Kritische Phänomene, Friedr. Vieweg \& Sohn, Braunschweig/Wisbaden (1980).

[23] D. H. Rischke, M. I. Gorenstein, H. Stöcker, and W. Greiner, Z. Phys. C 51, 485 (1991).

[24] L. D. Landau and E. M. Lifshitz, Statistical Physics (Course of Theoretical Physics, Volume 5), ButterworthHeinemann.

[25] P. Glansdorff and I. Prigogine, Thermodynamic Theory of Structure, Stability and Fluctuations, WileyInterscience, London, 1971.

[26] P. F. Kolb and U. Heinz, in Quark Gluon Plasma 3, R. C. Hwa and X. N. Wang (Eds.), World Scientific, Singapore, (nucl-th/0305084).

[27] J. D. Bjorken, Phys. Rev. D 27, 140 (1983).

[28] E. Brézin, J. C. Le Guillou, and J. Zinn-Justin, in Phase Transitions and Critical Phenomena, C. Domb and M. S. Green (Eds.) (Academic Press, New York, 1976), Vol. 6.

[29] B. Berdnikov and K. Rajagopal, Phys. Rev. D 61, 105017 (2000).

[30] P. C. Hohenberg and B. I. Halperin, Rev. Mod. Phys. 49,
435 (1977).

[31] D. T. Son and M. A. Stephanov, hep-ph/0401052

[32] M. Asakawa, T. Csörgö, and M. Gyulassy, Phys. Rev. Lett. 83, 4013 (1999).

[33] N. Xu and M. Kaneta, Nucl. Phys. A 698, 306c (2002); T. Chujo, for the PHENIX Collaboration, Nucl. Phys. A 715, 151c (2003); J. Adams et al. [STAR Collaboration], Phys. Rev. Lett. 92, 112301 (2004); A. Jipa, for the BRAHMS Collaboration, nucl-ex/0404011

[34] C. Roland et al. [NA49 Collaboration], nucl-ex/0403035 M. Kaneta [NA44 Collaboration], PhD thesis, Hiroshima University (1999); I. G. Bearden et al. [NA44 Collaboration] Phys. Rev. C 66, 044907 (2002).

[35] J. Sollfrank, U. Heinz, H. Sorge, and N. Xu, Phys. Rev. C 59, 1637 (1999).

[36] G. Van Buren, for the STAR Collaboration, Nucl. Phys. A 715, 129c (2003).

[37] A. Kiyomichi [PHENIX Collaboration], talk at DNP03 meeting in Tucson, U.S.A., November 1, 2003.

[38] CERES Collaboration, Nucl. Phys. A 727, 97 (2003).

[39] D. H. Rischke, Nucl. Phys. A 610, 88c (1996); D. H. Rischke, Y. Pürsün, J. A. Maruhn, H. Stöcker, and W. Greiner, Heavy Ion Phys. 1, 309 (1995).

[40] P. F. Kolb, Phys. Rev. C 68, 031902 (2003).

[41] C. Nonaka, E. Honda, and S. Muroya, Eur. Phys. J. C 17, 663 (2000).

[42] T. Hirano, Phys. Rev. Lett. 86, 2754 (2001); Phys. Rev. C 65, 011901 (2002).

[43] C. Nonaka, in progress.

[44] C. Bernard et al. [MILC Collaboration], Nucl. Phys. Proc. Suppl. 106, 429 (2002); Nucl. Phys. Proc. Suppl. 119, 523 (2003); Nucl. Phys. Proc. Suppl. 129, 626 (2004); hep-lat/0405029 